\title{
Nonlinear Consensus Control of UAV Elastic Formation
}

\author{
Guang-Yan XU ${ }^{1, a}$, Dan ZHAO 2,b, ${ }^{,}$, Hong-Mei ZHANG ${ }^{3, c}$ and Biao ZHOU ${ }^{4, d}$ \\ ${ }^{1,3}$ School of Aerospace Engineering, Shenyang Aerospace University, Shenyang, China, 110136 \\ 2, 4,School of Automation, Shenyang Aerospace University, Shenyang, China, 110136 \\ aguangyan_xu@163.com, 'bhaodan19900406@163.com, 'zhanghm001@yeah.net, \\ dzhoubiaoxt@163.com \\ ${ }^{*}$ Corresponding author
}

Keywords: Nonlinear control, Elastic formation, Consensus strategy, UUB

\begin{abstract}
The unmanned aerial vehicle (UAV) formation flight is studied by consensus strategy. Firstly, the UAV formation model with nonlinear elastic characteristics is established and proved to be Uniformly Ultimately Bounded (UUB). Secondly, a consensus strategy controller is designed combining with the proposed nonlinear elastic vector. Finally, a simulation is conducted to verify that the designed controller is effective and can reduce the fuel consumption and the control cost.
\end{abstract}

\section{Introduction}

At present, the consensus theory is widely used in the consensus of the complex network, multi-sensor, and formation keeping and so on. The consensus refers that each member in the group shares information, so that their status ultimately tends to be uniform [1-3].

An effective method based on decentralized control for the UAV formation with overlapping information flow is presented [4]. Behavior-based method makes the deviation vector tend to be consistent between every vehicle's true position and desired position. The direction of information flow is ring topologies of arbitrary direction, so as to realize the formation keeping [5]. A given virtual structure formation control scheme is studied to make the fixed wing UAV formation maneuver along the planned formation trajectory [6]. In this paper, nonlinear consensus strategy on formation keeping is imposed by leader-follower approach. The stabilization of the designed system is demonstrated by the presented UUB method.

\section{Nonlinear consensus formation model and control law design}

Formation model. The dynamic model of single UAV is:

$$
\begin{aligned}
& \dot{X}_{i}=v_{i} \cos \psi_{i} \\
& \dot{Y}_{i}=v_{i} \sin \psi_{i} \\
& v_{i} \dot{\psi}_{i}=b_{i} \quad, \quad i=1,2 \cdots n \\
& \dot{v}_{i}=a_{i}
\end{aligned} .
$$

where, $\left(X_{i}, Y_{i}\right)$ is the position of $i$-th $\mathrm{UAV}$ in the world coodinate. $v_{i}$ and $\psi_{i}$ are the velocity and yaw angle, respectively. $a_{i}$ and $b_{i}$ are tangential and normal accelerations respectively. 
The relative geometry position relationship between adjacent UAVs can be shown as Fig. $1 . P_{0}$ represents the leader UAV and $P_{i}$ represents the $i$-th UAV. $\bar{P}_{i}$ and $\hat{P}_{i}$ are the expected look-ahead point and the elastic look-ahead point of UAV $P_{i}$, respectively.

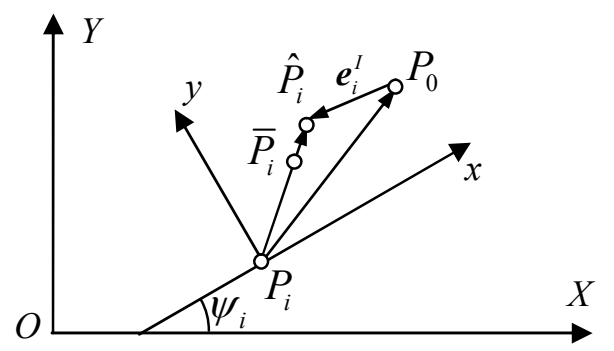

Fig. 1 Relative position relationship between two adjacent UAVs

Introduce the following nonlinear transformation

$$
\boldsymbol{z}_{i}=\left(\begin{array}{c}
\boldsymbol{z}_{i}^{I} \\
\boldsymbol{z}_{i}^{I I}
\end{array}\right), \quad \boldsymbol{z}_{i}^{I}=\left(\begin{array}{c}
X_{i} \\
Y_{i}
\end{array}\right), \quad \boldsymbol{z}_{i}^{I I}=\left(\begin{array}{c}
v_{i} \cos \psi_{i} \\
v_{i} \sin \psi_{i}
\end{array}\right), \quad \boldsymbol{u}_{i}=M_{i}^{-1} \boldsymbol{\eta}_{i}, \quad \boldsymbol{M}_{i}=\left(\begin{array}{cc}
\cos \psi_{i} & \sin \psi_{i} \\
-\frac{\sin \psi_{i}}{v_{i}} & \frac{\cos \psi_{i}}{v_{i}}
\end{array}\right) .
$$

Moreover, introduce a nonlinear elastic vector

$$
\hat{\boldsymbol{d}}_{i}=q\left(v_{i}\right) \overline{\boldsymbol{d}}_{i}=k\left(v_{d}, \overline{\boldsymbol{d}}_{i}\right) \cdot F\left(v_{i}\right) \hat{\overline{\boldsymbol{d}}}_{i} .
$$

with

$$
k\left(v_{d}, \overline{\boldsymbol{d}}_{i}\right)=\frac{\left|\overline{\boldsymbol{d}}_{i}\right|}{\sqrt{v_{d}}}, F\left(v_{i}\right)=\sqrt{v_{i}} .
$$

where, constant scalar $k\left(v_{d}, \overline{\boldsymbol{d}}_{i}\right)$ is similar to the elastic coefficient. The variable vector $F\left(v_{i}\right)$ is similar to the force. $\hat{\overline{\boldsymbol{d}}}_{i}$ is a unit vector of $\overline{\boldsymbol{d}}_{i}$ and represents the force direction. $\overline{\boldsymbol{d}}_{i}=\left(\bar{d}_{i}^{x}, \bar{d}_{i}^{y}\right)^{T}$ is the desired relative distance of adjacent UAVs. $v_{d}$ is the desired velocity.

According to the elastic look-ahead point $\hat{P}_{i}$

$$
\hat{P}_{i}=\boldsymbol{z}_{i}^{I}+\hat{\mathbf{d}}_{i}=\boldsymbol{z}_{i}^{I}+q\left(v_{i}\right) \overline{\boldsymbol{d}}_{i}=\boldsymbol{z}_{i}^{I}+k\left(v_{d}, \overline{\boldsymbol{d}}_{i}\right) \cdot F\left(v_{i}\right) \hat{\overline{\boldsymbol{d}}}_{i} .
$$

Differentiate (5) and the dynamic equations of single elastic front viewpoint are obtained.

$\dot{\hat{P}}_{i}=\boldsymbol{z}_{i}^{I I}+\dot{\hat{\mathbf{d}}}_{i}$

$\dot{\boldsymbol{z}}_{i}^{I I}=u_{i} \quad \forall i=1, . ., \mathrm{n}$

Define error vector $\boldsymbol{e}_{i}=\left(\boldsymbol{e}_{i}^{I}, \boldsymbol{e}_{i}^{I I}\right)^{T}$, and differentiate it to time $t$

$$
\begin{aligned}
\dot{\boldsymbol{e}}_{i}^{I} & =\left(\dot{\boldsymbol{z}}_{i}^{I}-\dot{\boldsymbol{z}}_{i-1}^{I}\right)+\dot{\hat{\boldsymbol{d}}}_{i} \quad i=\forall 2, . ., n . \\
\dot{\boldsymbol{e}}_{i}^{I I} & =\dot{\boldsymbol{z}}_{i}^{I I}-\dot{\boldsymbol{z}}_{i-1}^{I I}
\end{aligned}
$$

After the arithmetic, the error model is finally obtained 
$\dot{\boldsymbol{e}}_{i}^{I}=\boldsymbol{e}_{i}^{I I}+D_{i} W\left(\psi_{i}, v_{i}\right) \boldsymbol{u}_{i}$
$\dot{\boldsymbol{e}}_{i}^{I I}=\mathbf{u}_{i}-\mathbf{u}_{i-1}$, where $D_{i}=\left(\begin{array}{cc}\bar{d}_{i}^{x} & -\bar{d}_{i}^{y} \\ \bar{d}_{i}^{y} & \bar{d}_{i}^{x}\end{array}\right), W\left(\psi_{i}, v_{i}\right)=R\left(\psi_{i}\right)\left(\begin{array}{cc}\frac{\partial q\left(v_{i}\right)}{\partial v_{i}} & 0 \\ 0 & \frac{q\left(v_{i}\right)}{v_{i}}\end{array}\right) R^{T}\left(\psi_{i}\right)(8)$

Ignore $\mathbf{u}_{i-1}$, the equation (8) can be written as a linear time-varying system

$\dot{\mathbf{e}}_{i}=\left(\begin{array}{ll}0 & I \\ 0 & 0\end{array}\right) \dot{\mathbf{e}}_{i}+\left(\begin{array}{c}D_{i} W\left(\psi_{i}, v_{i}\right) \\ I\end{array}\right) \mathbf{u}_{i}=\mathrm{A} \dot{\mathbf{e}}_{i}+\mathrm{B}\left(\psi_{i}, v_{i}\right) \mathbf{u}_{i}$.

Design of control law. The consensus control law of UAV elastic formation is

$$
\boldsymbol{u}_{i}=-\sum_{j=1}^{n} g_{i j} k_{1 i}\left(\boldsymbol{e}_{i}-\boldsymbol{e}_{j}\right)-k_{2 i} \boldsymbol{e}_{i} \quad \forall i=1, . ., n
$$

where, $k_{1 i}$ and $k_{2 i}$ are the relative distance feedback gain and the system forward gain respectively. $\boldsymbol{e}_{i}$ and $\boldsymbol{e}_{j}$ are the state variable errors of the $i$-th and $j$-th UAVs. Then, the closed-loop control equation of the system under the information topology is derived as:

$$
\dot{\boldsymbol{e}}=\left(I_{N} \otimes \boldsymbol{A}-\left(K_{2}+K_{1}(L+G)\right) \otimes \bar{B}\right) \boldsymbol{e}-I_{N} \otimes \boldsymbol{C} \boldsymbol{u}_{0} .
$$

where, $K_{1}=\operatorname{diag}\left(k_{11} k_{12} \cdots k_{1 n}\right), K_{2}=\operatorname{diag}\left(k_{21} k_{22} \cdots k_{2 n}\right), \bar{B}=\boldsymbol{B}\left[\begin{array}{ll}I & 0\end{array}\right], \boldsymbol{e}=\operatorname{diag}\left(e_{1} e_{2} \cdots e_{n}\right)$, $L=\left[\sum_{j=1}^{n} g_{1 j},-g_{12} \cdots-g_{1 n} ; \ldots ;-g_{n 1},-g_{n 2} \cdots \sum_{j=1}^{n} g_{n j}\right], \quad G=\operatorname{diag}\left(-g_{11}-g_{22} \cdots-g_{n n}\right)$.

Put the relative value into (11) and the control variable can be obtained

$$
\boldsymbol{\eta}_{i}=-M_{i} \sum_{j=1}^{n}\left(g_{i j} k_{1 i}\left(\left(\mathbf{x}_{i}+\hat{\boldsymbol{d}}_{i}\right)-\left(\mathbf{x}_{j}+\hat{\boldsymbol{d}}_{j}\right)\right)-k_{2 i}\left(\left(\mathbf{x}_{i}+\hat{\boldsymbol{d}}_{i}\right)-\mathbf{x}_{0}\right)\right) .
$$

\section{UUB analysis of nonlinear consensus control}

Definition1. If there exists a non-negative bound $B^{m}(m=1, \ldots, M)$ and a finite time $T_{m}\left(B^{m}, \varepsilon^{1}\left(t_{0}\right), \cdots, \varepsilon^{M}\left(t_{0}\right)\right)$, so that $\left\|\varepsilon^{m}(t)\right\| \leq B^{m}, \forall t \geq t_{0}+T_{m}$. Then $\varepsilon^{m}$ is UUB.

Assumption1. The directed graph have a spanning tree and at least on root node.

Assumption2. The control input $\left\|\boldsymbol{u}_{i}\right\| \leq \mathbf{U}_{M}, \forall i=1, . ., n$, where, $\mathbf{U}_{M}$ is unknown and bounded.

Lemmal. Let $\bar{L}=K_{2}+K_{1}(L+G), \overline{\boldsymbol{B}}=\boldsymbol{B}\left[\begin{array}{ll}I & 0\end{array}\right]$ and $\lambda_{i}$ are eigenvalues of $\bar{L}$. Suppose the matrix $H_{i}=A-k \bar{B}$ is Hurwitz, the network topology graph $\mathrm{G}$ has a spanning tree and at least one root node, $k_{1 i}>0$ and $k_{2 i}>0$. if and only if the matrix $A-\lambda_{l} \boldsymbol{B}, \forall l$ is all Hurwitz, then 


$$
W=I_{N} \otimes \boldsymbol{A}-\bar{L} \otimes \overline{\boldsymbol{B}}
$$

is (31)Hurwitz.

Proof: If all $A-\lambda_{l} \boldsymbol{B}$ are Hurwitz, then matrix W is Hurwitz. Obviously, $\bar{L}=K_{2}+K_{1}(L+G)$ is satisfied with properties of Laplacian matrix that all of its eigenvalues are greater than zero. What's more, every entry of diagonal matrix $K_{1}$ is greater than zero. $k_{1 i} \lambda_{l}$ is greater than zero. Namely, $\lambda_{l}$ is greater than zero. So, all matrices $A-\lambda_{l} \overline{\boldsymbol{B}}$ are Hurwitz. Then, matrix W is Hurwitz.

Theorem1. Suppose Lemmal is satisfied. If the inequality

$$
\|\boldsymbol{e}\| \geq \frac{2 \bar{\sigma} P_{2} C U_{M}}{\beta}
$$

holds, the formation system (11) is UUB under controller (12). In inequality (14), $P_{2}$ is a positive definite matrix, $\bar{\sigma}$ and $\beta$ are real vectors.

Proof: Considering the candidate Lyapunov function

$$
V=\frac{1}{2} \boldsymbol{e}^{T} P_{2} \boldsymbol{e} .
$$

where, $p_{2}=p_{2}^{T}, p_{2}>0$ is a positive definite matrix. The control law $v$ of UAV formation is

$$
v=-c\left(I_{n} \otimes K_{i 1}\right) \boldsymbol{e} .
$$

From Assumption 1, $k_{i 2} \neq 0, L+G$ is irreversible. Put(15) into(16). Then the derivative of it is

$$
\dot{V}=\frac{1}{2}\left[W \boldsymbol{e}-C \boldsymbol{u}_{0}\right]^{T} P_{2} \boldsymbol{e}+\frac{1}{2} \boldsymbol{e}^{T} P_{2}\left[W \boldsymbol{e}-C \boldsymbol{u}_{0}\right]=\frac{1}{2} \boldsymbol{e}^{T}\left(W^{T} P_{2}+P_{2} W\right) \boldsymbol{e}-\frac{1}{2}\left(\boldsymbol{e}^{T} P_{2} C \boldsymbol{u}_{0}+\boldsymbol{u}_{0}^{T} C^{T} P_{2} \boldsymbol{e}\right) .
$$

For $\boldsymbol{e}^{T} P_{2} C \boldsymbol{u}_{0}$ and $\boldsymbol{u}_{0}{ }^{T} C^{T} P_{2} \boldsymbol{e}$ are all scalars, then $\boldsymbol{u}_{0}^{T} C^{T} P_{2} \boldsymbol{e}=\left[\boldsymbol{u}_{0}{ }^{T} C^{T} P_{2} \boldsymbol{e}\right]^{T}=\boldsymbol{e}^{T} P_{2} C \boldsymbol{u}_{0}$. From Lemma1, $W$ is Hurwitz asymptotically stable, and it meets $P_{2} W+W^{T} P_{2}=-\beta I_{N r}$. So

$$
\dot{V}=\frac{-\beta}{2}\|\boldsymbol{e}\|^{2}-\boldsymbol{e}^{T} P_{2} C \boldsymbol{u} .
$$

According to the Assumption 2

$$
\dot{V} \leq-\frac{\beta}{2}\|\boldsymbol{e}\|^{2}+\|\boldsymbol{e}\| \bar{\sigma} P_{2} C U_{M}
$$

To make $\dot{V} \leq 0$, only the following inequality needs to meet

$$
\|\boldsymbol{e}\| \geq \frac{2 \bar{\sigma} P_{2} C U_{M}}{\beta} \text {. }
$$

As shown in Definition 1, the formation error vector $\boldsymbol{e}$ is UUB.

\section{Simulations}

A 3-UAV formation is simulated. The simulation time is $70 \mathrm{~s}$. The desired formation speed is $\bar{v}_{\mathrm{d}}=50 \mathrm{~m} / \mathrm{s}$. The desired relative distance of adjacent UAVs are $\bar{d}_{2}^{x}=50, \bar{d}_{3}^{x}=100, \bar{d}_{2}^{y}=100$, $\bar{d}_{3}^{y}=200$. The initial location are UAV1 $(200,200), \operatorname{UAV} 2(100,150), \operatorname{UAV} 3(0,100)$. 


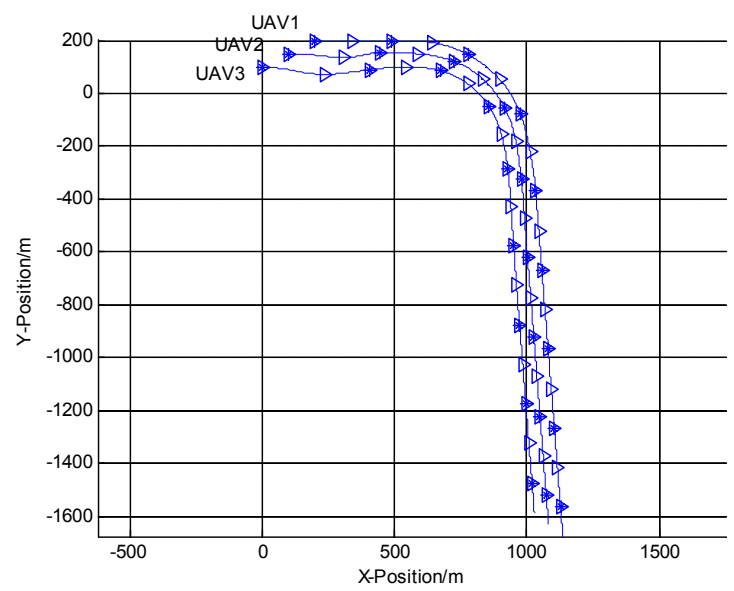

Fig. 2 UAV formation flight trajectory of nonlinear consensus control

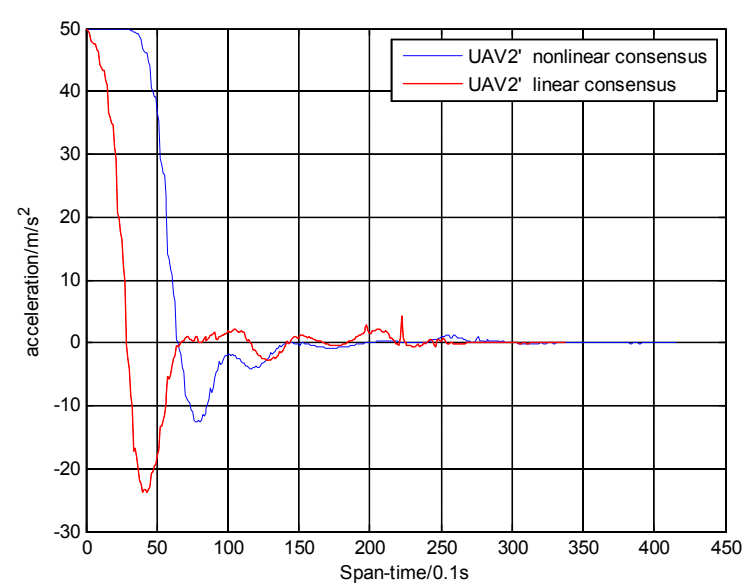

Fig. 3 the angular velocities of UAV2 between nonlinear and linear consensus strategy $\left(v_{d}=50 \mathrm{~m} / \mathrm{s}\right)$

From the formation flight trajectory in Fig. 2, we can see that formation keeping can be realized with the nonlinear consensus control method. Fig. 3 shows the comparison of nonlinear consensus and linear consensus control strategies. The control law curve of nonlinear consensus controller has a lower cost and smaller overshoot than the linear consensus controller. When maneuvering, the nonlinear consensus controller has better formation keeping control effect. The advantage of UUB control is shown.

\section{Conclusions}

UUB control of UAV nonlinear elastic formation consensus strategy is deeply discussed. The control effect of the nonlinear elastic consensus controller is verified. With the comparison of the nonlinear and the linear consensus control strategies, the former has a better control effect and less cost. It has the obvious advantages over the latter.

\section{Acknowledgement}

This research was financially supported by the National Science Foundation of China (61074159).

\section{References}

[1] R. Olfati-Saber, R. M. Murray, Consensus problems in networks of agents with switching topology and time-delays, IEEE Trans. Autom. Control. 49 (2004) 1520-1533.

[2] W. Ren, R. W. Beard, Consensus seeking in multi-agent systems under dynamically changing interaction topologies, IEEE Trans. Autom. Control. 50 (2005) 655-661.

[3] W. Ren, Y. Cao, Distributed coordination of multi-agent net-works, Springer-Verlag, London, 2011.

[4] D. M. Stipanovic, G. Inalhan, R. Teo, et al, Decentralized overlapping control of a formation of unmanned aerial vehicles, Automatica, 40 (2004), 1285-1296.

[5] J. R. Lawton, R. W. Beard and B. Young, A decentralized approach to formation maneuvers, IEEE Trans. Robot. Autom., 19 (2003), 933-941.

[6] C. B. Low, A dynamic virtual structure formation control for fixed-wing UAVs, 20119 th IEEE International Conference on Control and Automation (ICCA) Santiago, Chile, (2011), 19-21. 